\title{
Gamma-ray polarization constraints on Planck scale violations of special relativity
}

\author{
Luca Maccione* \\ DESY, Theory Group, Notkestrasse 85, D-22607 Hamburg (Germany) \\ II. Institut für Theoretische Physik, Universität Hamburg, Luruper Chaussee 149, D-22761 \\ Hamburg (Germany) \\ E-mail: luca.maccione@desy.de
}

\section{Stefano Liberati}

SISSA, Via Beirut 2-4, I-34014 Trieste, Italy

INFN, Via Valerio 2, I-34127 Trieste, Italy

E-mail: liberatiesissa.it

\section{Annalisa Celotti}

SISSA, Via Beirut 2-4, I-34014 Trieste, Italy

INFN, Via Valerio 2, I-34127 Trieste, Italy

E-mail: celotti@sissa.it

\section{John G. Kirk}

Max-PIanck-Institute für KernPhysik, Saupfercheckweg 1, D-69117 Heidelberg, Germany

E-mail: john.kirk@mpi-hd.mpg.de

\section{Pietro Ubertini}

IASF-INAF, via Fosso del Cavaliere 100, Roma, Italy

E-mail: pietro.ubertini@iasf-roma.inaf.it

We exploit recent polarimetric observations of the Crab Nebula in the hard X-ray band by INTEGRAL to show that the absence of vacuum birefringence effects constrains $O(E / M)$ Lorentz violation in QED to the level $|\xi|<9 \times 10^{-10}$ (at $3 \sigma \mathrm{CL}$ ). This tightens by more than three orders of magnitude previous constraints. We also show that planned X-ray polarimeters have the potential to probe $|\xi| \sim 10^{-16}$ by detecting polarization in active galaxies at red-shift $\sim 1$.

Polarimetry days in Rome: Crab status, theory and prospects

October 16-17, 2008

Rome, Italy

\footnotetext{
${ }^{*}$ Speaker.
} 


\section{Introduction}

Recent years have witnessed a growing interest in the possible high energy violations of local Lorentz Invariance (LI) as well as a flourishing of observational tests. Indeed, specific hints of Lorentz invariance violations (LV) arose from various approaches to Quantum Gravity (QG, see $[1,2]$ for detailed references).

In most of the above mentioned QG models, LV enters through dispersion relations which can be cast in the general form (it is assumed, for simplicity, that rotational invariance is preserved and only boost invariance is affected by Planck-scale corrections):

$$
E^{2}=p^{2}+m^{2}+f(E, p ; \mu ; M)
$$

where we set the low energy speed of light $c=1, E$ and $p$ are the particle energy and momentum, $\mu$ is a particle-physics mass-scale (possibly associated with a symmetry breaking/emergence scale) and $M$ denotes the relevant QG scale. Generally, it is assumed that $M$ is of order the Planck mass: $M \sim M_{\mathrm{Pl}} \approx 1.22 \times 10^{19} \mathrm{GeV}$, corresponding to a quantum (or emergent) gravity effect. The function $f(E, p ; \mu ; M)$ can be expanded in powers of the momentum (energy and momentum are basically indistinguishable at high energies, although they are both taken to be smaller than the Planck scale), and the lowest order LV terms ( $p, p^{2}$ and $p^{3}$ ) have been mainly considered [1].

At first sight, it appears hopeless to search for effects suppressed by the Planck scale. Even the most energetic particles ever detected (Ultra High Energy Cosmic Rays) have $E \lesssim 10^{11} \mathrm{GeV} \sim$ $10^{-8} M_{\mathrm{Pl}}$. However, even tiny corrections can be magnified into a significant effect when dealing with high energies (but still well below the Planck scale), long distances of signal propagation, or peculiar reactions (see, e.g., [1]). A partial list of these windows on quantum gravity includes:

- sidereal variation of LV couplings as the lab moves with respect to a preferred frame or direction

- cumulative effects: long baseline dispersion and vacuum birefringence (e.g. of signals from gamma ray bursts, active galactic nuclei, pulsars)

- anomalous (normally forbidden) threshold reactions allowed by LV terms (e.g. photon decay, vacuum Čerenkov effect)

- shifting of existing threshold reactions (e.g. photon annihilation from blazars, GZK reaction)

- LV induced decays not characterised by a threshold (e.g. decay of a particle from one helicity to the other or photon splitting)

- maximum velocity (e.g. synchrotron peak from supernova remnants)

- dynamical effects of LV background fields (e.g. gravitational coupling and additional wave modes)

However, most of these effects require a well established theoretical framework to calculate reaction rates and describe the particle dynamics. Although we study here a purely kinematic effect, we prefer for definiteness to work within the framework of Effective Field Theory with 
non-renormalizable, mass dimension $5 \mathrm{LV}$ operators (see [1,3] and references therein) restricted to QED. In this framework the most general dispersion relations for photons and electrons are

$$
\begin{aligned}
& \omega_{ \pm}^{2}=k^{2} \pm \xi k^{3} / M_{\mathrm{Pl}} \\
& E_{ \pm}^{2}=p^{2}+m^{2}+\eta_{ \pm} p^{3} / M_{\mathrm{Pl}},
\end{aligned}
$$

where (1.2) refers to photons ${ }^{1}$ and (1.3) to fermions ${ }^{2}$. The constants $\xi$ and $\eta_{ \pm}$indicate the strength of the LV. The \pm signs denote right and left circular polarization in (1.2), and positive and negative helicity states of the fermion in (1.3). Equation (1.2) implies that the direction of polarization rotates during propagation due to the different velocities of the right- and left-handed circular polarizations, $\mathrm{v}_{ \pm} \simeq 1 \pm \xi k / M$. This effect is known as vacuum birefringence (VB).

Recently $\eta_{ \pm}$have been constrained to have a magnitude less than $10^{-5}$ at $95 \%$ confidence level (CL) by a detailed analysis of the synchrotron component of the Crab Nebula (CN) broadband spectrum [5], while the constraint $|\xi| \lesssim 2 \times 10^{-7}$ has been obtained by [6] considering the absence of VB effects during the propagation of optical/UV polarized light from Gamma-Ray Bursts (GRB). There are also preliminary indications, based on an analysis of the photon fraction in Ultra-High-Energy Cosmic Rays, that these coefficients might be less than $10^{-14}[7,8,9]$.

In this contribution, we describe how the current constraints on $O\left(E / M_{\mathrm{Pl}}\right)$ suppressed LV can be further tightened by about three orders of magnitude for photons [10], by considering the limits on VB effects implied by the recently detected [11] polarized hard X-rays from the CN. Firstly, we set such constraints following the arguments by $[12,13]$. This approach is robust against systematic uncertainties related to astrophysical modeling. We then infer tighter limits that exploit and rely on modeling of the Crab Nebula and pulsar.

Note that this LV term violates CPT symmetry. Studies of higher order CPT conserving terms would be extremely important (see [2]). However, they cannot be constrained by the methods used here because they do not predict vacuum birefringence.

Finally, we discuss the constraints which future X-ray polarization measurements of extragalactic objects, e.g. Active Galactic Nuclei (AGN) will allow. This is of particular interest in the light of current experimental efforts to build X-ray polarimeters [14, 15, 16, 17, 18, 19].

\section{Birefringence}

During propagation over a distance $d^{3}$, the polarization vector of a linearly polarized plane wave with momentum $k$ rotates through an angle [4, 12, 13, 2],

$$
\theta(k, d)=\frac{\omega_{+}(k)-\omega_{-}(k)}{2} d \simeq \xi \frac{k^{2} d}{2 M_{\mathrm{Pl}}} .
$$

Depending on the available information on both the observational and the theoretical (i.e. source modeling) side, observations of polarized light from a distant source can constrain $|\xi|$ in two ways.

\footnotetext{
${ }^{1}$ This kind of dispersion relation was also derived in some semi-classical limit of Loop quantum gravity [4].

${ }^{2}$ For positrons we have $\eta_{ \pm}^{\text {pos }}=-\eta_{\mp}^{\text {el }}[2]$.

${ }^{3}$ For an extragalactic object at redshift $z$, the (cosmological) distance is given by $d(z)=\frac{1}{H_{0}} \int_{0}^{z} \frac{1+z^{\prime}}{\sqrt{\Omega_{\Lambda}+\Omega_{m}\left(1+z^{\prime}\right)^{3}}} d z^{\prime}$, which includes a $\left(1+z^{\prime}\right)^{2}$ factor in the integrand to take into account the red-shift acting on the photon energies. As usual, $H_{0}$ is the present value of the Hubble parameter, while $\Omega_{\Lambda}$ and $\Omega_{m}$ represent the density fractions of cosmological constant and matter in the Universe, respectively.
} 
Decrease in polarization degree Since detectors have a finite energy bandwidth, eq. (2.1) is never probed in real situations. Rather, if some net amount of polarization is measured in the band $k_{1}<E<k_{2}$, an order-of-magnitude constraint arises from the fact that if the angle of polarization rotation (2.1) were to differ by more than $\pi / 2$ over this band, the detected polarization would fluctuate sufficiently for the net signal polarization to be suppressed [12, 13]. From (2.1), this constraint is

$$
\xi \lesssim \frac{\pi M_{\mathrm{Pl}}}{\left(k_{2}^{2}-k_{1}^{2}\right) d(z)}
$$

This just requires that any intrinsic polarization (at source) is not completely washed out during signal propagation. It thus relies on the mere detection of a polarized signal, without considering the observed polarization degree. A more refined limit can be obtained by calculating the maximum observable polarization degree, given the maximum intrinsic value [20]:

$$
\Pi(\xi)=\Pi(0) \sqrt{\langle\cos (2 \theta)\rangle_{\mathscr{P}}^{2}+\langle\sin (2 \theta)\rangle_{\mathscr{P}}^{2}},
$$

where $\Pi(0)$ is the maximum intrinsic degree of polarization, $\theta$ is defined in eq. (2.1) and the average is weighted over the source spectrum and instrumental efficiency, represented by the normalized weight function $\mathscr{P}(k)$ [12]. Conservatively, one can set $\Pi(0)=100 \%$, but a lower value can sometimes be justified on the basis of source modeling. Using eq. (2.3), one can then cast a constraint by requiring $\Pi(\xi)$ to exceed the observed value.

Rotation of polarization angle Suppose that polarized light measured in a certain energy band has a position angle $\theta_{\mathrm{obs}}$ with respect to a fixed direction. At fixed energy, the polarization vector rotates by the angle $(2.1)^{4}$; if the position angle is measured by averaging over a certain energy range, the final net rotation $\langle\Delta \theta\rangle$ is given by the superposition of the polarization vectors of all the photons in that range:

$$
\tan (2\langle\Delta \theta\rangle)=\frac{\langle\sin (2 \theta)\rangle_{\mathscr{P}}}{\langle\cos (2 \theta)\rangle_{\mathscr{P}}}
$$

where $\theta$ is given by (2.1). If the position angle at emission $\theta_{\mathrm{i}}$ in the same energy band is known from a model of the emitting source, a constraint can be set by imposing

$$
\tan (2\langle\Delta \theta\rangle)<\tan \left(2 \theta_{\mathrm{obs}}-2 \theta_{\mathrm{i}}\right)
$$

Although this limit is tighter than that obtained from the previous methods, it clearly hinges on assumptions about the nature of the source, which may introduce significant uncertainties.

\section{Constraints from the Crab nebula}

In the case of the $\mathrm{CN}, \mathrm{a}(46 \pm 10) \%$ degree of linear polarization in the $100 \mathrm{keV}-1 \mathrm{MeV}$ band has recently been measured by the INTEGRAL mission [21, 11] (see also [22]). This measurement

\footnotetext{
${ }^{4}$ Faraday rotation is negligible at such energies.
} 
uses all photons within the SPI instrument energy band. However the convolution of the instrumental sensitivity to polarization with the detected number counts as a function of energy, $\mathscr{P}(k)$, is maximized and approximately constant within a narrower energy band (150 to $300 \mathrm{keV})$ and falls steeply outside this range [23]. Hence we shall, conservatively, assume that most polarized photons are concentrated in this band. Given $d_{\text {Crab }}=1.9 \mathrm{kpc}, k_{2}=300 \mathrm{keV}$ and $k_{1}=150 \mathrm{keV}$, eq. (2.2) leads to the order-of-magnitude estimate $|\xi| \lesssim 2 \times 10^{-9}$. A more accurate limit follows from (2.3). In the case of the $\mathrm{CN}$ there is a robust understanding that photons in the range of interest are produced via the synchrotron process, for which the maximum degree of intrinsic linear polarization is about $70 \%$ (see e.g. [24]). The requirement $\Pi(\xi)>16 \%$ (taking account of a $3 \sigma$ offset from the best fit value $46 \%$ ) leads to the constraint (at 99\% CL) $|\xi| \lesssim 6 \times 10^{-9}$ [10]. It is interesting to notice that X-ray polarization measurements of the $\mathrm{CN}$ already available in 1978 [25], set a constraint $|\xi| \lesssim 5.4 \times 10^{-6}$, only one order of magnitude less stringent than that reported in [6].

This constraint can be tightened by exploiting the current astrophysical understanding of the source. The $\mathrm{CN}$ is a cloud of relativistic particles and fields powered by a rapidly rotating, strongly magnetized neutron star. Both the Hubble Space Telescope and the Chandra X-ray satellite have imaged the system, revealing a jet and torus that clearly identify the neutron star rotation axis [26]. The projection of this axis on the sky lies at a position angle of $124.0^{\circ} \pm 0.1^{\circ}$ (measured from North in anti-clockwise). The neutron star itself emits pulsed radiation at its rotation frequency of $30 \mathrm{~Hz}$. In the optical band these pulses are superimposed on a fainter steady component with a linear polarization degree of $30 \%$ and direction precisely aligned with that of the rotation axis [27]. The direction of polarization measured by INTEGRAL-SPI in the $\gamma$-rays is $\theta_{\mathrm{obs}}=123^{\circ} \pm 11^{\circ}(1 \sigma$ error) from the North, thus also closely aligned with the jet direction and remarkably consistent with the optical observations. This compelling (theoretical and observational) evidence allows us to use eq. (2.5). Conservatively assuming $\theta_{\mathrm{i}}-\theta_{\mathrm{obs}}=33^{\circ}$ (i.e. $3 \sigma$ from $\theta_{\mathrm{i}}, 99 \% \mathrm{CL}$ ), this translates into the limit $|\xi| \lesssim 9 \times 10^{-10}[10]$.

\section{Discussion}

The constraints presented in section 3 are remarkably strong. Although based on a cumulative effect, they are achieved using a local (Galactic) object. The reason lies, on the one hand, in the quadratic dependence of $\theta$ on the photon energy, in constrast with the linear gain given by distance (see e.g. eq. (2.1)). On the other hand, the robust theoretical understanding of the $\mathrm{CN}$ has enabled us to strengthen the constraints significantly.

Further improvements on LV constraints via birefringenge are expected thanks to the forthcoming high-energy polarimeters, which will provide an unprecedented sensitivity, sufficient to detect polarized light at a few \% levels also in extragalactic sources. The LV limits will be optimized by balancing between source distance and observational energy range depending on the detector sensitivity as shown in [10]. Remarkably, constraints of order $|\xi|<O\left(10^{-16}\right)$ could be placed if some polarized distant sources $(z \sim 1)$ will be observed by such instruments at $1 \mathrm{MeV}$.

\section{Acknowledgments}

LM acknowledges support from SISSA where most part of this work was done. 


\section{References}

[1] D. Mattingly, Living Rev. Rel. 8 (2005) 5 [arXiv:gr-qc/0502097].

[2] T. Jacobson, S. Liberati and D. Mattingly, Annals Phys. 321 (2006) 150 [arXiv:astro-ph/0505267].

[3] R. C. Myers and M. Pospelov, Phys. Rev. Lett. 90 (2003) 211601 [arXiv:hep-ph/0301124].

[4] R. Gambini and J. Pullin, Phys. Rev. D 59 (1999) 124021 [arXiv:gr-qc/9809038].

[5] L. Maccione, S. Liberati, A. Celotti and J. G. Kirk, JCAP 0710 (2007) 013 [arXiv:0707.2673 [astro-ph]].

[6] Y. Z. Fan, D. M. Wei and D. Xu, Mon. Not. Roy. Astron. Soc. 376 (2006) 1857 [arXiv:astro-ph/0702006].

[7] M. Galaverni and G. Sigl, Phys. Rev. Lett. 100 (2008) 021102 [arXiv:0708.1737 [astro-ph]].

[8] L. Maccione and S. Liberati, JCAP 0808 (2008) 027 [arXiv:0805.2548 [astro-ph]].

[9] M. Galaverni and G. Sigl, Phys. Rev. D 78 (2008) 063003 [arXiv:0807.1210 [astro-ph]].

[10] L. Maccione, S. Liberati, A. Celotti, J. G. Kirk and P. Ubertini, Phys. Rev. D 78 (2008) 103003 [arXiv:0809.0220 [astro-ph]].

[11] A. J. Dean et al., Science 321 (2008) 1183.

[12] R. J. Gleiser and C. N. Kozameh, Phys. Rev. D 64 (2001) 083007 [arXiv:gr-qc/0102093].

[13] T. A. Jacobson, S. Liberati, D. Mattingly and F. W. Stecker, Phys. Rev. Lett. 93 (2004) 021101 [arXiv:astro-ph/0309681].

[14] E. Costa et al., arXiv:astro-ph/0609576.

[15] F. Muleri et al., arXiv:astro-ph/0609573.

[16] Y. Kanai et al., Nucl. Instrum. Meth. A 570, 61 (2007).

[17] E. Costa et al., arXiv:astro-ph/0207440.

[18] J. Knodlseder, P. Von Ballmoos, F. Frontera, A. Bazzano, F. E. Christensen, M. Hernanz and C. Wunderer, arXiv:0707.4627 [astro-ph].

[19] H. Krawczynski, Polarimetry with EXIST, same conference

[20] McMaster, W. H. 1961, Reviews of Modern Physics, 33, 8

[21] Parmar, A. N., Winkler, C., Barr, P., Hansson, L., Kuulkers, E., Much, R., \& Orr, A. 2003, Proceedings of the SPIE, 4851, 1104

[22] M. Forot, P. Laurent, I. A. Grenier, C. Gouiffes and F. Lebrun, arXiv:0809.1292 [astro-ph].

[23] S. McGlynn et al., arXiv:astro-ph/0702738.

[24] J. Petri and J. G. Kirk, Astrophys. J. 627 (2005) L37 [arXiv:astro-ph/0505427].

[25] Weisskopf, M. C., Silver, E. H., Kestenbaum, H. L., Long, K. S., \& Novick, R. 1978, Astrophys. J. Letters, 220, L117

[26] C. Y. Ng and R. W. Romani, Astrophys. J. 601 (2004) 479 [arXiv:astro-ph/0310155].

[27] G. Kanbach, A. Slowikowska, S. Kellner and H. Steinle, AIP Conf. Proc. 801, 306 (2005) [arXiv:astro-ph/0511636]. 